\title{
Animating Chinese Landscape Paintings and Panorama Using Multi-Perspective Modeling
}

\author{
Nelson Siu-Hang Chu Chiew-Lan Tai \\ Department of Computer Science \\ The Hong Kong University of Science and Technology \\ Clearwater Bay, Kowloon, Hong Kong \\ Email: \{cpegnel,taicl\}@ust.hk
}

\begin{abstract}
This paper describes a multi-perspective modeling technique for making fly-through animations from a single large landscape painting or panorama. These images have sub-scenes that are taken from different perspective views. The technique constructs a simple global model for the entire input image and builds a local model for each subscene using the TIP spidery mesh interface. Animation is generated by switching smoothly between a local model and the global model while moving a virtual camera along an animation path. Novel views are rendered by mapping the texture images, extracted from the original image, onto the active model. The usefulness of the technique is demonstrated with two animation examples from a Chinese landscape painting and a spherical panoramic image.
\end{abstract}

\section{Introduction}

Traditionally, two-dimensional (2D) transformation techniques have been used to add special effects to static scenes and shapes in images. Comparatively, making visually $3 \mathrm{D}$ animations from a single image is more difficult even to a skilled animator as this requires building 3D models. Computer vision techniques [12] are of not much help for extracting 3D models from a single image. Tedious trial-and-error manual approaches are thus needed to build models that closely resemble the scene depicted in the picture.

Horry et al. [5] showed that constructing only a rough 3D model of a scene could be sufficient for producing visually convincing $3 \mathrm{D}$ animations from some images. Their Tour Into the Picture (TIP) technique provides an intuitive and convenient interface for constructing a "box" model, consisting of at most five rectangles, for the background scene of an image. The foreground objects in the scene are modeled as polygonal "billboards", each is attached to at least one background rectangle. A drawback of the TIP technique is that the movement of the virtual camera is limited to a single box; thus, the animations tend to be too short (usually about 10 seconds) to be highly entertaining despite its convincing $3 \mathrm{D}$ effect.

In this paper, we extend the TIP technique to animate pictures that have higher space complexity - pictures that contain multiple sub-scenes, each being regarded as taken from a different perspective view. Such scenes are very common in Chinese landscape paintings. The original TIP technique cannot handle such images; it works well only for photographs or paintings that communicate a narrower view seen from one location. Traditional Chinese paintings are known to depict nature as not being observed simply from one particular location, although they generally appear as what would be seen by human eye from a considerable distance and altitude [13]. In fact, traditional Chinese hand-scroll landscape paintings aimed at taking the viewer through the artist's mind while the scroll is viewed section by section. A model that assumes only one viewing location cannot represent such a scene. Hence, we propose the multi-perspective TIP technique to animate these images.

With multi-perspective TIP, longer and more entertaining animations can be generated. The technique is also applicable to panoramic images $[2,11]$ to produce animations that have stronger $3 \mathrm{D}$ effect than those created by only panning and zooming from a fixed viewpoint.

\section{Related Work}

Image-based modeling and rendering (IBMR) has emerged as an attractive means for producing stunningly realistic novel views of scenes from a set of reference images, without constructing the geometric models. IBMR techniques range from making use of a single panoramic image $[2,11]$ to a $7 \mathrm{D}$ plenoptic function [7]. Rendering with plenoptic functions of fewer dimensions $[4,6,10]$ are devised by constraining the camera position or assuming limited depth of objects. Some techniques do not require any depth information, while others requires 


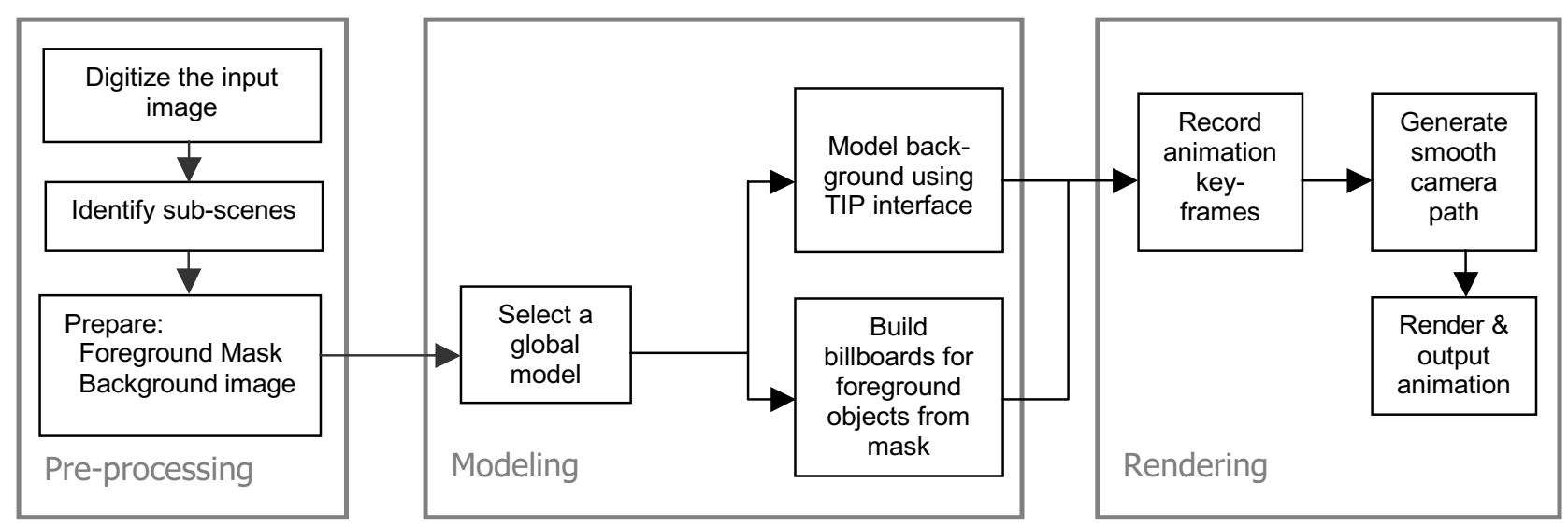

Figure 1. Overview of an animation system based on multi-perspective TIP

recovering of camera position and orientation [9] to determine the depth information.

Panorama viewers allow user to interactively look around an environment that is captured on a single panoramic image. However, the effect is limited to panning, zooming, and tilting from a fixed viewpoint. Concentric mosaic [10] effectively adds the horizontal parallax effect to ordinary panorama, thus giving a much better user experience to virtual navigation. A limitation is that the viewpoint and the viewing direction are constrained to lie in a circular region on a plane; thus it cannot produce fly-through animations, which often give better visual effect than walk-through animations for outdoor and landscape scenes.

Most IBMR techniques deal with photographs or synthesized images. The TIP technique is one of the few that can generate novel views from paintings or drawings. The TIP technique [5] allows viewers to "tour into" the scene of a single image. Fleishman et al. [3] attempted to use the TIP technique to link several sparse views that are taken along a specific camera path. This approach removes the problem of limited touring space of the TIP technique, but the image correspondence problem was not solved satisfactorily to produce good walk-through animations.

\section{Overview of Multi-perspective TIP}

The multi-perspective TIP can be divided into three phases: pre-processing phase, modeling phase, and rendering phase. Figure 1 shows an overview of an animation system based on multi-perspective TIP. In the pre-processing phase, the user identifies, from an input image, sub-scenes that can be regarded as separate perspective views. Each sub-scene corresponds to a 3D space into which the user can tour. Next, like in the TIP technique, the user decides which objects are foreground objects. These objects should lie inside the identified subscenes. Two new images, the foreground mask and the background image (Figure 2), which in effect distinguish the foreground objects from the background scene, are then prepared from the input image using commercial software such as Photoshop. With interactive segmentation techniques such as intelligent scissors [8] implemented as lasso tools in commercial software, it is not difficult to segment the foreground objects. The background image is obtained by erasing the foreground objects and patching the missing background details using tools like "clone brush" in commercial software.

For modelling the scene, the user first selects a global model for the entire image. It is a rectangle, cylinder, or sphere, depending on whether the input image is a painting, cylindrical or spherical panorama, respectively. The user then constructs a box-like local model for each sub-scene using the TIP spidery mesh interface (Figure 2(d)) and models the foreground objects in each sub-scene as polygons attached to some background rectangle [5].

In the rendering phase, as the virtual camera moves, its location determines which geometric model (local or global) is active, and the active model is texture-mapped
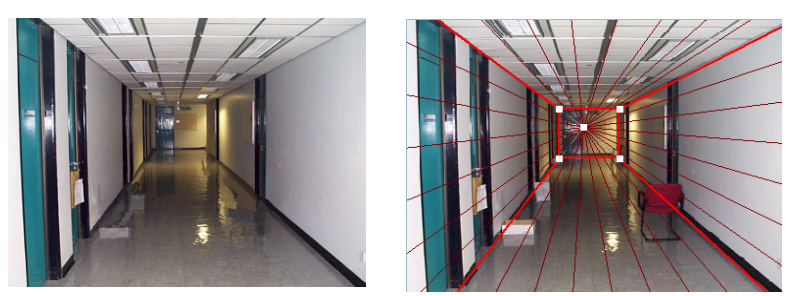

$\begin{array}{ll}\text { (a) a scene } & \text { (b) foreground mask }\end{array}$
Foreground Objects

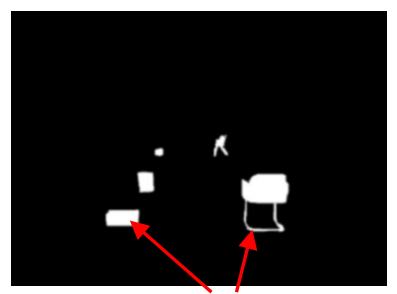

(c) background image

(d) spidery mesh

Figure 2. Input image, supplementary images, and the spidery mesh 
to produce a new view. Viewing parameters are specified by the user at each keyframe and are interpolated for the in-between frames.

Since the pre-processing phase is similar to the original TIP, we shall only discuss the modeling and rendering phases in details in the following two sections. We shall first consider the case of animating landscape paintings, and leave the discussion on panoramic input to Section 7.

\section{Modeling}

Suppose there are $n$ sub-scenes identified in the preprocessing phase. In the modeling phrase, a simple global model $M^{g}$ is selected for the entire image, and a local model $M_{i}^{l}$ is constructed for the $i$ th sub-scene. Billboards standing in 3D space are also created for the foreground objects in each sub-scene.

\subsection{Global modeling for entire input image}

For paintings, the global model $M^{g}$ is simply a rectangle (Figure 3(a)). Its texture map is thus the entire input image. We define a global coordinate system $F_{0}$ such that the rectangle is on the plane $z=0$, with its left and bottom edges along the $x$ and $y$ axes of $F_{0}$, respectively.

\subsection{Local modeling of sub-scenes}

Using the TIP spidery mesh, the user specifies a vanishing point and an inner rectangle on each sub-scene image [5]. (We assume one-point perspective scene. Discussion on two-point and three-point perspective can be found in [1].) The inner rectangle and the spidery edges prescribe the boundaries of (at most) five rectangles of the local model $M_{i}^{l}$ : floor, ceiling, left wall, right wall, and rear wall. To compute the $3 \mathrm{D}$ vertices of $M_{i}^{l}$, we make the following assumptions on the initial viewing parameters, which will produce the sub-scene image as a perspective view. We define a local coordinate system $F_{i}$ for sub-scene $i$ such that the image projection plane is the

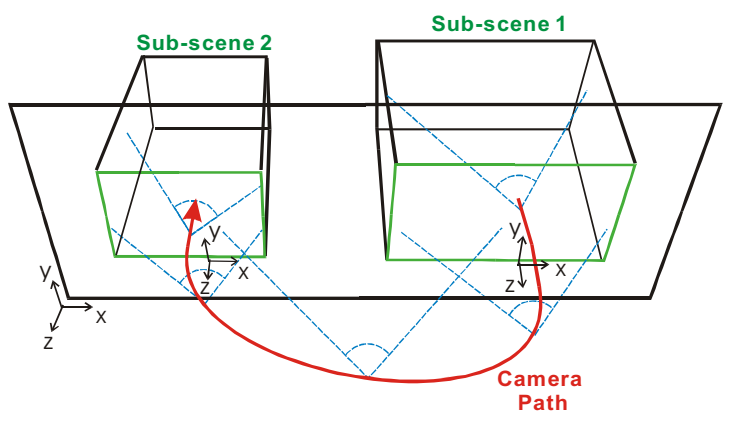

(a) Rectilinear input (perspective view) plane $z=0$, the view up vector is $(0,1,0)$, the floor is on the plane $y=0$, and the viewing vector from the initial viewpoint $\boldsymbol{v}_{i}$ (in the direction of the specified vanishing point) is in the negative $z$ direction lying on the plane $x=0$ (Figure 3(a)).

The definitions of the coordinate systems serve to facilitate transformations between the global and local models. Let $\boldsymbol{O}_{i}$ denote the coordinates (relative to the global frame $F_{0}$ ) of the origin of $F_{i}$. Since the axes of all the frames are oriented in the same directions, the change of coordinates between the frames can be expressed in terms of only translations and scalings; the scalings can be further avoided by using the same scale across all the frames. With these settings, the change of coordinates from frame $F_{i}$ to $F_{0}$ can be simply expressed as $\boldsymbol{p}\left[F_{0}\right]=$ $\boldsymbol{p}\left[F_{i}\right]+\boldsymbol{O}_{i}$, where $\boldsymbol{p}[F]$ denotes the coordinates of a point $\boldsymbol{p}$ relative to frame $F$. Conversely, when switching from a global model to a local model, we only need to compute $\boldsymbol{p}\left[F_{i}\right]=\boldsymbol{p}\left[F_{o}\right]-\boldsymbol{O}_{i}$.

Each foreground object is modeled as a "billboard" that is attached to at least one of the background rectangles [5]. Horry et al. proposed using hierarchical polygonal models to achieve more realistic rendering of complex foreground objects. This approach, however, requires the user to manually specify the bounding polygons of the objects. Favoring an automatic method for identifying the boundaries of foreground objects from the foreground mask and for extracting their texture maps, we decide to model each foreground object simply as a rectangle. We find this trading of realistic rendering of complex objects for production speed to be not a problem for animating most images. With this specification, the $3 \mathrm{D}$ coordinates of the foreground rectangles and the texture maps can be determined by simply scanning the foreground mask line by line. When a "white" (i.e., the grey-scale value is above a user-defined threshold) pixel is encountered, the "white" region to which the pixel belongs is then flood-filled. While flood-filling, the color of the corresponding pixel in the original image and the alpha value in the mask are extracted to be the RGBA value of the texture map of that foreground object. At the same time, the bounding

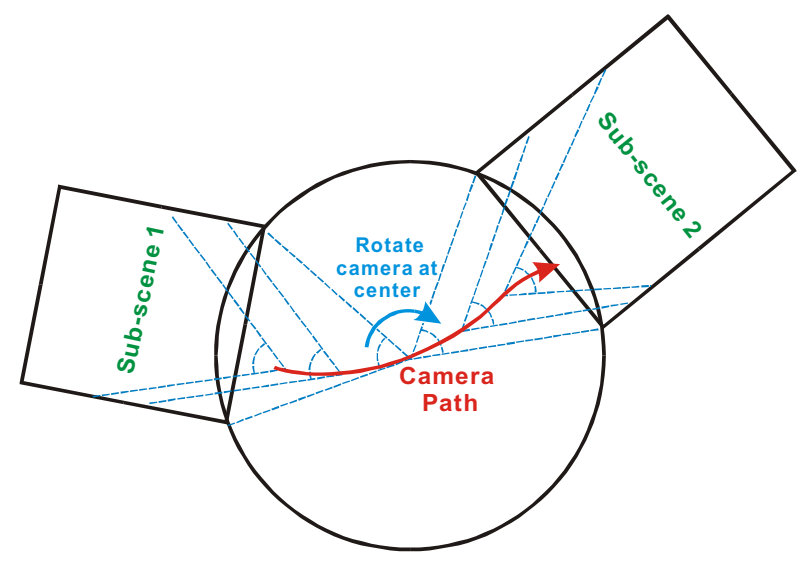

(b) Cylindrical or spherical panoramic input (top view)

Figure 3. A camera path from one sub-scene to another 
rectangle of that foreground object, in pixel coordinates, is determined for calculating its 3D coordinates once we know to which background rectangle it is attached. After the entire mask is scanned, every disjoint foreground object would be identified. If two objects of different depths overlap and appear as one object in the mask, we solve this problem by drawing a 1-pixel-wide dark line between them in the mask. On a $300 \mathrm{MHz}$ Pentium-III PC, it takes about 5 seconds to perform the foreground modeling for the 4800x1600 landscape painting in Figure 5(a).

\section{Rendering and Making Animations}

The viewing location determines whether a local model or the global model is active. When a local model is active, the touring effect is similar to the TIP technique; when the global model is active, a projected view of a part of the input image is seen.

Our graphical user interface allows the user to specify the viewing position and direction for each keyframe. The viewing positions are interpolated by a $C^{2}$ cubic spline to obtain a smooth camera path. To ensure smooth switching between the models when touring into and out from subscene $i$, we include in the animation path the initial viewpoint $\boldsymbol{v}_{i}$. In addition to these viewing parameters, the user can prescribe a viewing window for each sub-scene, all with the same aspect ratio. The field-of-view at $\boldsymbol{v}_{i}$ is then computed from the viewing window. If no viewing window is specified, we assume that the field-of-view is fixed throughout the animation, and define it to be the maximal intersection of the initial frustums of all the subscenes.

Another constraint for achieving smooth animation is

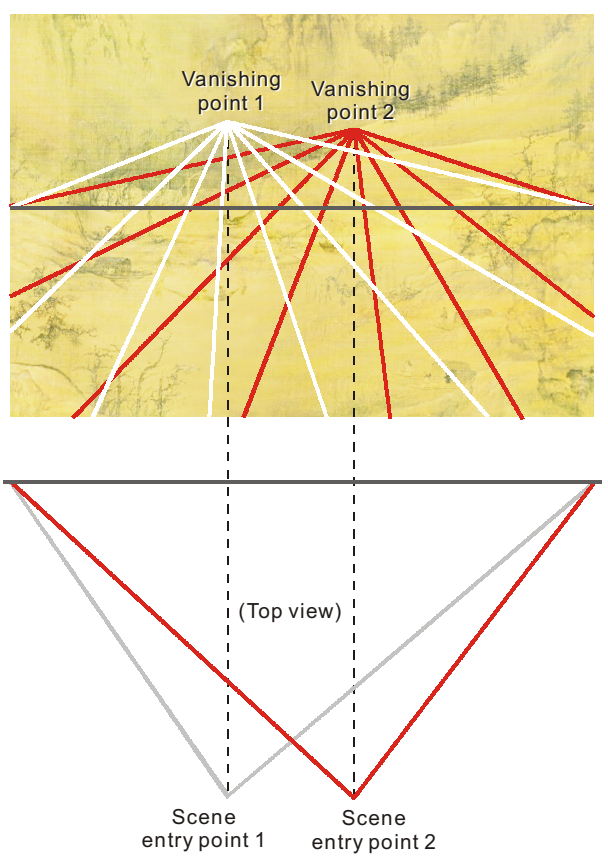

Figure 4. Different scene entry points resulted from different vanishing points that the viewing direction must return to be along the negative $\mathrm{z}$-axis at a scene entry point so that the rendered view is just a projected view of the original image. Thus, we insert an additional keyframe, with constrained viewing parameters, at each entry point to ensure smooth scene transition.

Recall that the initial viewpoint is determined by the vanishing point of the sub-scene. For landscape paintings, the user often has much leeway in specifying the vanishing point, thus the user can take into account the desired entry point of animation when specifying the vanishing point. Figure 4 shows how a different scene entry point can be obtained by changing the vanishing point.

During the animation, a local model $M_{i}^{l}$ is active if the camera is inside $M_{i}^{l}$, or outside it but within the corresponding initial viewing frustum. The global model $M^{g}$ is active if none of the local models is active.

The viewing window also serves another purpose: it clips away the portion of the sub-scene near the scene boundary that often appear distorted from certain viewpoints, especially for large foreground objects that extend to the scene boundary.

\section{Implementation and An Example}

We have implemented an animation system based on the proposed multi-perspective TIP technique. The system is written in Object Pascal using Borland Delphi 4 and runs on the MS-Windows platform.

We perform a perspective warping of the extracted textures prior to rendering so that we can treat the corrected textures as normal texture maps and make use of the OpenGL texture mapping routines. Real-time scene navigation is possible for input images of 800x600 pixels and an output size of $400 \times 300$ pixels on a $233 \mathrm{MHz}$ Pentium-II PC without an OpenGL accelerated graphics card. With acceleration, real-time walk-through of images of higher resolutions, with anti-aliased texturing, can be achieved.

As an example, we apply our technique to tour an eleventh-century Chinese landscape painting entitled Fishing village in mild snow ${ }^{l}$. Figure 6(a) shows a portion of the original painting. Two sub-scenes are identified in this portion. Notice that it is not possible to tour into this painting using the original TIP because the horizons of the two sub-scenes are at different levels; even if the subscenes have the same horizon, a single TIP box is insufficient for modelling the two cavities corresponding to the sub-scenes. This animation lasts about 90 seconds.

As Chinese landscape painting is an art of time and space, which provides an opportunity not only for viewing but also for taking a spiritual journey [13], we have demonstrated that multi-perspective TIP technique provides a means to appreciate this journey in an explicit way.

\footnotetext{
${ }^{1}$ A hand-scroll by Wang Shen (1036-?) in the Sòng dynasty. The original painting is more then two times wider.
} 


\section{Touring into Panoramic Images}

The proposed technique is also applicable to panoramic images. For cylindrical panorama, the global model is a cylinder; for spherical panorama, it is a sphere (Figure 3 (b)). The input image is first mapped to the global model to present an undistorted image to the user to identify the sub-scenes. Each sub-scene is then projected onto a plane to perform perspective modeling using the TIP spidery mesh. The user can tour into a local model, return to the centre of the global model, pan to another sub-scene and tour into its local model, and so on; that is, instead of panning and zooming from a fixed viewpoint allowed by traditional panorama viewing, with multi-perspective TIP, the user can move the viewpoint into the local models to produce fly-through animations of higher immersed feeling.

Figure 6 shows a spherical panoramic image that is prepared from two photographs taken with a fish-eye lens. Figure 6 (b)-(d) and (h)-(k) are some rendered views of a local model, and (e)-(g) are rendered views when the global spherical model is active. This animation example was made in half an hour, including the pre-processing and rendering time. The animation files are available at http: //www. cs. ust.hk/ cpegnel/ACLP/.

\section{Conclusions and Future Work}

We have presented a method for making fly-through animations from a single image consisting of multiple perspective views. Our method can be regarded as an extension to the original TIP technique. We simplify the specification of the foreground objects to automate that foreground modeling process from the foreground mask and thus speed-up the production time.

Most IBMR techniques use a single geometric model, complex or simple, to represent a given scene. In this paper, we show that more than one model can be built for one scene, and switching of models can be carried out during the animation.

The technique has the limitation that the animation path is constrained to return to the initial viewing parameters of each sub-scene before touring into another sub-scene. This could limit its use in certain navigation applications, however the technique allows the user to quickly produce visually convincing $3 \mathrm{D}$ animation from landscape paintings and panorama, which cannot be achieved with previous techniques.

It would be interesting to extend the animation system to handle video sequence so that a user can navigate in a dynamic scene. One advantage of having a dynamic scene is that we can recover the background pixel information for the parts that are occluded by a moving foreground object. Furthermore, by assuming a fixed camera position, simple motion tracking techniques may be able to automatically yield the foreground mask.

\section{Acknowledgments}

Many thanks go to Man-Tak Fong and Yiu-Cho Leung for their help in implementing the animation system and gathering sample input images. The authors are also grateful to the Publishing Technology Centre of HKUST for providing the fish-eye lens photographs.

\section{References}

[1] K. Anjyo and Y. Horry. Theory and Practice of "Tour Into the Picture", SIGGRAPH 98 Course notes \#8, 1998.

[2] S.E. Chen. QuickTime VR - An image-based approach to virtual environment navigation. In SIGGRAPH 95 Conference Proceedings, pages 29-38, August 1995.

[3] S. Fleishman, B. Chen, A. Kaufman, and D. Cohen-Or. Navigating through sparse views. Symposium on Virtual Reality Software and Technology '99.

[4] S. J. Gortler, R. G., R. Szeliski, and M. F. Cohen. The lumigraph. In SIGGRAPH 96 Conference Proceedings, pages 43-54, August 1996.

[5] Y. Horry, K.I Anjyo, and K. Arai. Tour Into the Picture: Using a spidery mesh interface to make animation from a single image. In SIGGRAPH 97 Conference Proceedings, pages 225-232, August 1997.

[6] M. Levoy and P. Hanrahan. Light field rendering. In SIGGRAPH 96 Conference Proceedings, pages 31-42, August 1996.

[7] L. McMillan and G. Bishop. Plenoptic modeling: An imagebased rendering system. In SIGGRAPH 95 Conference Proceedings, pages 39-46, August 1995.

[8] E. N. Mortensen and W.A. Barrett. Intelligent scissors for image composition. In SIGGRAPH 95 Conference Proceedings, pages 191--198, August 1995.

[9] S. M. Seitz and C. R. Dyer. View morphing. In SIGGRAPH 96 Conference Proceedings, pages 21-30, August 1996.

[10] H. Y. Shum and L. W. He. Rendering with concentric mosaics. In SIGGRAPH 99 Conference Proceedings, pages 299-306, August 1999.

[11] R. Szeliski and H. Y. Shum. Creating full view panoramic mosaics and environment maps. In SIGGRAPH 95 Conference Proceedings, pages 39-46, August 1995.

[12] E. Trucco and A. Verri. Introductory techniques for $3 D$ computer vision, Prentice Hall, 1998.

[13] W. Wong. The tao of Chinese landscape painting: principles and methods. Design Press, New York, 1990. 

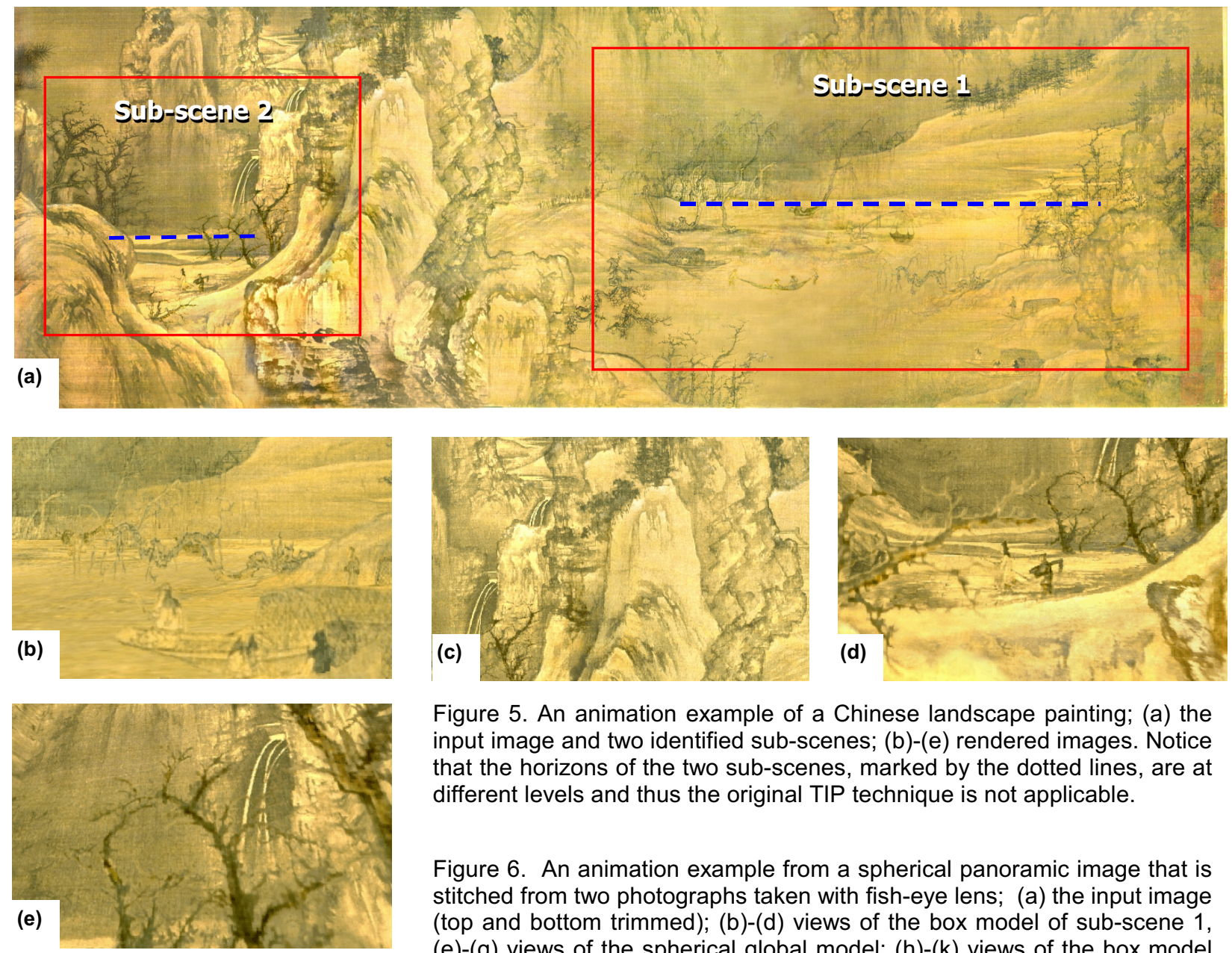

Figure 5. An animation example of a Chinese landscape painting; (a) the input image and two identified sub-scenes; (b)-(e) rendered images. Notice that the horizons of the two sub-scenes, marked by the dotted lines, are at different levels and thus the original TIP technique is not applicable.

Figure 6. An animation example from a spherical panoramic image that is stitched from two photographs taken with fish-eye lens; (a) the input image (top and bottom trimmed); (b)-(d) views of the box model of sub-scene 1, (e)-(g) views of the spherical global model; $(\mathrm{h})-(\mathrm{k})$ views of the box model of sub-scene 2 .
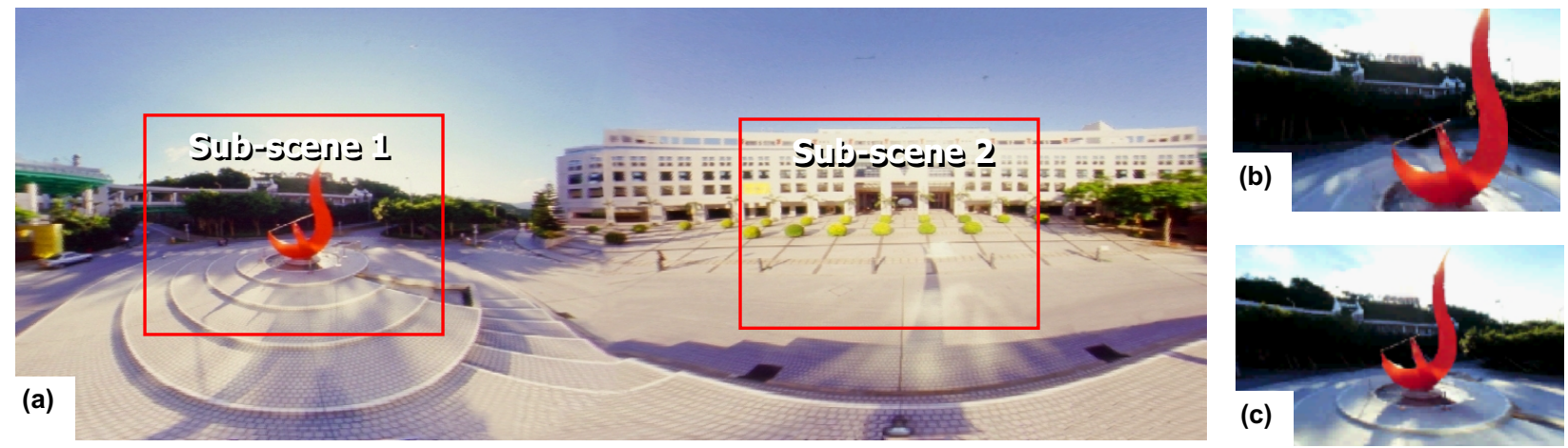
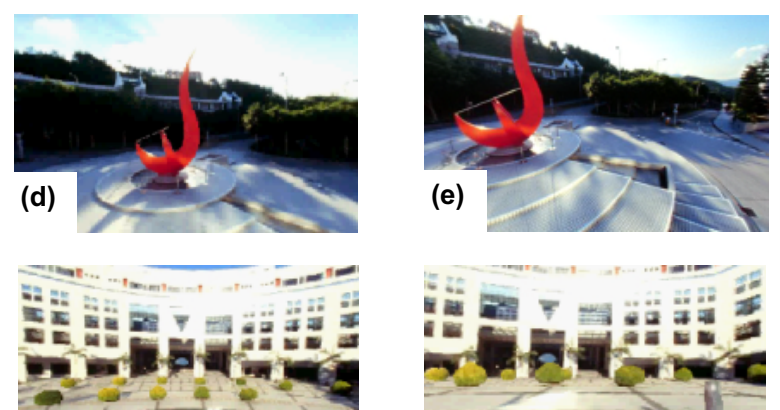

(h)

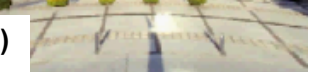

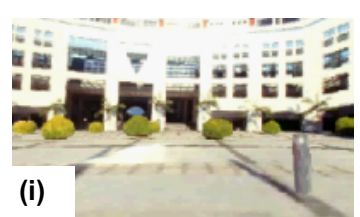
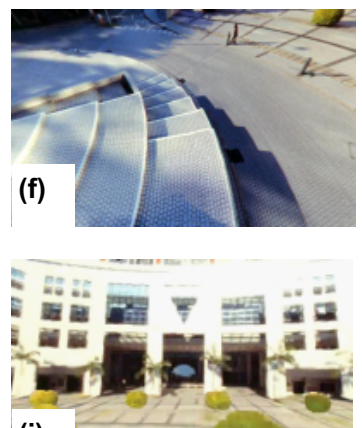

(j)

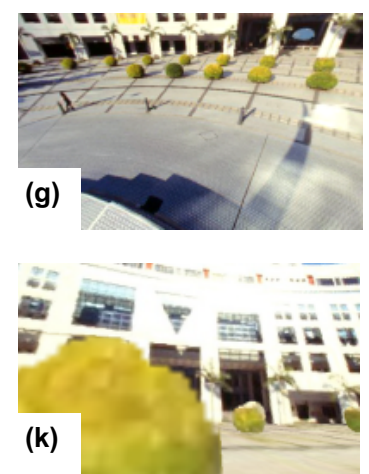

